\title{
LA IDENTIFICACIÓN DE HABILIDADES Y \\ ESTRATEGIAS DE ESCRITURA DE ESTUDIANTES \\ DE POSGRADO NO HISPANOHABLANTES A TRAVÉS DE ENTREVISTAS SEMIESTRUCTURADAS
}

\author{
THE IDENTIFICATION OF WRITING SKILLS AND STRATEGIES \\ OF NON-NATIVE SPANISH-SPEAKING GRADUATE STUDENTS \\ THROUGH SEMI-STRUCTURED INTERVIEWS
}

\author{
ARTURO MENDOZA \\ Universidad Nacional Autónoma de México, Ciudad de México, México. \\ a.mendoza@enallt.unam.mx
}

\section{RESUMEN}

La escritura académica representa una habilidad indispensable para el éxito académico. En el caso de los estudiantes no nativo hablantes, el dominio de dicha habilidad implica una dificultad adicional: el uso eficiente de la lengua meta. Por este motivo, resulta necesario evaluar si estos prospectos universitarios cuentan con un dominio suficiente de la lengua meta. De ahí que el objetivo del presente estudio fuera indagar cuáles eran las habilidades y estrategias de escritura de estudiantes de posgrado no hispano hablantes que habían sustentado un examen de español con fines académicos. El estudio consistió en entrevistar a 25 estudiantes de posgrado respecto de sus habilidades y estrategias de escritura. Estas habilidades fueron evaluadas mediante una rúbrica analítica, y con base en los resultados, los estudiantes fueron agrupados en tres niveles de desempeño: intermedio bajo, intermedio alto y avanzado. Posteriormente, las entrevistas fueron analizadas en su contenido por dos codificadores mediante un enfoque tipo inductivo. Los resultados cualitativos sugieren que los estudiantes del grupo intermedio bajo carecen de habilidades y estrategias de escritura para el ámbito académico, mientras que los del grupo intermedio alto y avanzado cuentan con un nivel de escritura apto para el posgrado.

Palabras clave: Escritura académica en L2, habilidades y estrategias de escritura, exámenes de español con fines académicos, extrapolación en exámenes de lengua.

\section{ABSTRACT}

Writing skills at the graduate and undergraduate level are necessary for academic success. In the case of non-native speaking students, the mastery of academic writing skills encompasses an additional difficulty: the proficient use of the target language. For this 
reason, it is necessary to assess whether these future university students meet the necessary threshold level in the target language. Hence, the aim of this study was to investigate the writing skills and strategies of non-native Spanish-speaking graduate students that sat a Spanish test for academic purposes. 25 graduate students were interviewed regarding their writing skills and strategies. These skills were assessed by means of an analytic rating scale, and based on the results, students were grouped into three performance levels: lowintermediate, high-intermediate and advanced. Afterwards, the content of the interviews was analyzed by two coders following an inductive approach. The qualitative results suggest that students at the low-intermediate level lack the minimum writing skills and strategies required in an academic setting, whereas those at the high-intermediate and the advanced groups reveal suitable writing skills at the graduate level.

Keywords: Academic writing in L2, writing skills and strategies, Spanish language tests for academic purposes, extrapolation in language tests.

Recibido: 10.10.2017. Aceptado: 05.01.2018.

\section{INTRODUCCIÓN}

T a escritura en el contexto universitario representa una de las habilidades primordiales con que deben contar los estudiantes para ser académicamente competentes (Carlino, 2003, 2013; Cassany, 2006; Cassany y Morales, 2008). De hecho, la escritura representa una de las prácticas más comunes que emplean los docentes para evaluar el conocimiento a lo largo o al final de un ciclo escolar (Castelló, 2014; González, 2014; Mendoza, 2014). Sin embargo, el dominio de esta habilidad requiere de un aprendizaje formal y explícito a través de las distintas disciplinas académicas y ciclos universitarios (i.e., licenciatura, maestría y doctorado) (Carlino, 2013; Castelló e Iñesta, 2012; Flores, 2014). Conforme más avanzan los estudiantes en sus estudios de grado y de posgrado, mayores son las exigencias de dominio de escritura requeridas y, por lo tanto, las dificultades que encaran para escribir eficientemente en una amplia gama de géneros discursivos (Zhizhko, 2014). En el caso de los estudiantes universitarios no nativo hablantes, la dificultad de escribir de acuerdo con las convenciones formales y propias de cada uno de los géneros discursivos se ve acrecentada por las limitantes del dominio de la lengua meta (Leki, Cumming y Silva, 2008; Sasaki, 2014; Weigle, 2002). Con el fin de responder adecuadamente a la necesidad de escribir eficazmente en contextos académicos, los estudiantes despliegan una serie de estrategias que les facilitarán el proceso de redacción, revisión y edición de sus textos. Estas estrategias varían de estudiante en estudiante, pero en gran medida se relacionan con el uso de recursos alternativos para redactar (i.e., diccionarios, traductores y correctores ortográficos) y para editar sus textos (i.e., ayuda de terceros) (Singh, 2017). Adicionalmente, los estudiantes buscan ayuda de terce- 
ros u oportunidades de contacto con el uso de la lengua escrita (p.ej., tomar clases en el posgrado, cursos de lengua o de redacción) (Fox, 2004).

Ahora bien, con el fin de garantizar que los estudiantes universitarios cuentan con el nivel umbral de dominio de la lengua meta, es común que las universidades soliciten que sus prospectos universitarios estudiantes sustenten un examen de lengua con fines académicos. Estos exámenes son considerados de alto impacto dadas las implicaciones y consecuencias que conllevan en la vida de los examinados (Bachman y Palmer, 2010; Kane, 2013). Esto quiere decir que los estudiantes que no cuentan con un nivel mínimo de competencia comunicativa en la lengua meta, podrían ser rechazados u obligados a tomar cursos de lengua previos a su carga curricular ordinaria (Fox, 2004). Por esta razón, se debe garantizar que los exámenes funcionan adecuadamente y que las autoridades cuentan con información detallada, respecto del desempeño de los examinados, que les permita tomar decisiones justas y equitativas (Kane, 2013; Xi, 2010).

Sin embargo, dadas las implicaciones de los exámenes de alto impacto, la validación interna de los mismos resulta insuficiente. Por este motivo, dentro del proceso de diseño y validación de un examen de alto impacto, Kane, Crooks y Cohen (1999) proponen una serie de inferencias que deberán ser respaldadas mediante evidencia empírica para conferirle validez al examen. Una de estas inferencias es la de la extrapolación; es decir, es necesario demostrar hasta qué punto los exámenes son extrapolables al "mundo real" (Kane, 1992, p. 527). En el caso de la escritura, por ejemplo, se requiere de estudios que respalden que los estudiantes que demuestran un nivel aceptable en la prueba escrita (de ahora en adelante, PE) de un examen de certificación de lengua con fines académicos serán capaces de escribir de forma eficiente en el contexto universitario, y viceversa (Bachman y Palmer, 1996, 2010; Fulcher, 2010; Kane, 2002, 2006, 2013). En el caso del contexto anglosajón, los estudios de extrapolación son vastos, y recientemente se ha buscado extrapolar los resultados de un examen mediante entrevistas y cuestionarios (véase p.ej., Fox, 2004 y Weigle, 2010). Los hallazgos de Fox (2004) revelaron, por ejemplo, que el proceso de aculturación, la actitud, la motivación (reflejada en la asistencia a clase, la participación y el cumplimiento de tareas) y la ayuda de terceros juegan un papel importante en el desarrollo de habilidades comunicativas de los estudiantes no nativo hablantes en el ámbito académico. Sin lugar a dudas, la ventaja de contar con exámenes de lengua estandarizados (p.ej., TOEFL o IELTS) administrados ampliamente alrededor del mundo ha facilitado que se conduzcan estudios en este sector.

No obstante, en el caso del español, la falta de Exámenes de Certificación de Español para Ámbitos Académicos (ECEAA) administrados a gran escala ha dificultado que se lleven a cabo estudios de extrapolación. Adicionalmente, la gran mayoría de estudios que se han conducido recientemente en contextos universitarios hispanos es para saber cómo escriben los estudiantes hispanos en español 
(p.ej., Angulo, 2013; Cassany y Morales, 2008, Carciu, 2009; Hewitt y Felices, 2010) o en inglés (p.ej., Encinas y Keranen, 2010; Leki, Cumming y Silva, 2008), pero no para indagar cuáles son las habilidades o estrategias de escritura de los estudiantes no nativo hablantes del español. En la escritura en L2, a diferencia de la escritura en L1, existen dos componentes independientes, aunque interrelacionados entre sí, que inciden directamente en el proceso de escritura: el dominio de la segunda lengua y el dominio de las habilidades de escritura (Weigle, 2002). Por último, cabe señalar que en el caso particular de la escritura, no existe aún teoría alguna sobre el proceso de escritura en lengua extranjera (Knoch, 2011; Weigle, 2002), y los modelos existentes generalmente se enfocan en el proceso de escritura en lengua materna desde el punto de vista cognitivo (Bereiter y Scardamalia, 1987; Hayes, 1996; Hayes y Flower, 1980; Kellog, 1996) o sociocognitivo (Chang, Wu y Weir, 2014; Weir, 2005).

Por este motivo, en el contexto de los ECEAA, hacen falta estudios que permitan esclarecer la relación que existe entre el desempeño en un examen y el uso de la lengua en un contexto universitario. Dada la importancia de la escritura para la construcción del conocimiento en el ámbito académico, resultan particularmente indispensables los estudios que permitan extrapolar los resultados obtenidos en la PE de un ECEAA al dominio de la escritura dentro del contexto universitario.

\subsection{Las entrevistas semiestructuradas}

Las entrevistas, según Turner (2010), "proveen información a profundidad relacionada con las experiencias de los participantes, así como sus puntos de vista sobre un tema particular" (p. 134). También son consideradas como la manera más natural y socialmente aceptada de recolectar información relacionada con las experiencias de las personas, sus percepciones, actitudes o sentimientos con respecto a cierta temática (Dörnyei, 2007; Zhang y Wildemuth, 2009, p. 222). A diferencia de las entrevistas estructuradas (cerradas y poco flexibles para abundar en conceptos emergente) o abiertas (no delimitadas por un guion ni por preguntas de investigación acotadas), en las entrevistas semiestructuradas el investigador cuenta con una serie de preguntas abiertas predeterminadas en un guion (Mann, 2016). En este sentido, el tema que se desea explorar debe haber sido estudiado previamente para poder diseñar dicho guion. Sin embargo, el guion no es exhaustivo y debe ser lo suficientemente flexible para incorporar otros aspectos que emerjan durante las entrevistas (Mann, 2016, p. 102). Las entrevistas semiestructuradas, pese a que cuentan con un guion previamente establecido, son flexibles y permiten que el investigador profundice más en un aspecto relevante o interesante para su investigación (Tracy, 2013). De igual manera, es posible que nuevos temas o conceptos emerjan durante ellas. Naturalmente, debido a que se cuenta con un guion 
flexible, no es tan fácil que sean operadas por personas que se encuentran fuera del proceso de investigación (Zhang y Wildemuth, 2009). En cuanto al idioma en el cual se conduce la entrevista, Mann (2016) menciona que se debe poner especial cuidado cuando esta se lleva a cabo en una lengua diferente del entrevistador o entrevistado. Por último, de acuerdo con Riach (2009) el reto del investigador no solo reside en conducir la entrevista, sino también en la manera en la que conduce el análisis de los datos y se presentan los hallazgos en distintos niveles.

\subsection{Antecedentes y objetivo de la investigación}

Con el fin de garantizar una evaluación justa y equitativa para sus prospectos universitarios no hispanohablantes, la Universidad Nacional Autónoma de México decidió impulsar la creación de un nuevo examen de español como lengua extranjera para el ámbito académico (de ahora en adelante, EXELEAA). La razón de dicho cambio se debió a tres motivos fundamentales: 1) el examen anterior (Examen de Posesión de la Lengua Española) no fue diseñado con fines académicos, 2) el examen anterior no contaba con una prueba de respuesta construida para evaluar la expresión escrita, habilidad esencial en el ámbito académico (Mendoza, 2014, 2015a) y 3) no existen exámenes de español con fines académicos que sean administrados a gran escala (Vine y Ferreira, 2016; Mendoza y Knoch, 2018). Con el advenimiento del nuevo examen, sin embargo, también se presentó la necesidad de validarlo. Dado que el examen anterior no contaba con una PE, se consideró esencial conducir estudios relacionados con esta habilidad (véase Mendoza, 2015b). Siguiendo el enfoque basado en la argumentación para la validación de exámenes (Kane, 2002, 2006, 2013), se establecieron una serie de inferencias que requirieron ser respaldadas mediante evidencia empírica para poder justificar la confiabilidad y pertinencia de la PE del EXELEAA. Una de estas inferencias consistió en indagar hasta qué grado se podía extrapolar el resultado obtenido por los estudiantes en la PE del EXELEAA al dominio de su escritura académica. La siguiente pregunta de investigación que se formuló fue la siguiente:

¿Son extrapolables los resultados de la PE del EXELEAA a las habilidades de escritura de los estudiantes de posgrado no hispanohablantes?

\section{MÉTODOS}

\subsection{Los participantes}

En la entrevista participaron voluntariamente 25 estudiantes no hispanohablantes de posgrado que habían sustentado el EXELEAA ese mismo día. 


\subsection{La entrevista semiestructurada}

Con base en las propuestas de Fox (2004) y de Weigle (2010) para extrapolar los resultados de un examen de inglés con fines académicos, en la presente investigación se optó por utilizar una entrevista semiestructurada. Siguiendo la línea de Krippendorff (2004) y Lindlof y Taylor (2011), se diseñó un guion de entrevista semiestructurada partiendo de ciertas variables que se presuponía se encontraban asociadas al proceso de escritura académica. La entrevista la condujo el investigador en español, experto en la enseñanza y evaluación del español como lengua extranjera, con lo cual se mitigaron posibles efectos perniciosos al formular preguntas con vocabulario y estructuras gramaticales asequibles a los entrevistados (Mann, 2016; Zhang y Wildemuth, 2009). En caso de que los entrevistados presentaran problemas léxicos, sintácticos o gramaticales, el entrevistador proporcionó el apoyo necesario para que el entrevistado pudiera expresarse (Riach, 2009). En todo caso, los estudiantes contaban con un nivel de expresión oral suficiente para poder expresarse en español, aunque fuera con dificultades. Posteriormente, se llevó a cabo un pilotaje con cinco estudiantes de posgrado. Más adelante, un grupo de cuatro colegas y una especialista en análisis de entrevistas revisaron la categorización y, a partir de ahí, se afinó y amplió el guion de la entrevista (ver Anexo 1). En 2014 se aplicó la entrevista a otros 20 estudiantes que participaron en el segundo pilotaje del EXELEAA.

\subsection{La división de estudiantes en grupos}

Con el fin de evidenciar hasta qué punto eran extrapolables los resultados de la PE del EXELEAA al contexto de uso de la lengua escrita en el ámbito académico, la responsable del examen y el investigador diseñaron una rúbrica analítica para evaluar las habilidades y estrategias de escritura académica descritas por los estudiantes en la entrevista (véase Anexo 2). En esta rúbrica se incluyeron 12 categorías con cuatro bandas respectivamente. Con el fin de evaluar el funcionamiento de la rúbrica, la responsable del examen y el investigador evaluaron de forma independiente cinco entrevistas seleccionadas al azar. Tras el pilotaje, se revisaron los resultados y se llevaron a cabo modificaciones a la rúbrica. Posteriormente se evaluó a los otros 20 entrevistados. La correlación de Pearson entre los dos evaluadores fue de 0,86 . En caso de discrepancia de más de un $10 \%$, intervino un tercer evaluador ajeno a la investigación y se obtuvo la media de los 3 evaluadores. De las 25 entrevistas, 8 tuvieron que ser evaluadas nuevamente y solamente 1 presentó una discrepancia mayor al $20 \%$. De acuerdo con las puntuaciones obtenidas 
en la evaluación de la entrevista, los estudiantes fueron clasificados en 3 grupos: intermedio bajo, intermedio alto y avanzado. La Tabla I muestra la distribución de los estudiantes en grupos de acuerdo con el resultado obtenido en la entrevista. La correlación de Pearson entre ambos resultados fue de 0,72 , lo cual mostró una fuerte relación entre los resultados obtenidos en la PE y en la entrevista.

Tabla I. Agrupación de estudiantes entrevistados de acuerdo con el puntaje obtenido en la entrevista.

\begin{tabular}{|c|c|c|c|}
\hline Grupo & Estudiante & Nacionalidad & $\begin{array}{l}\text { Puntaje obtenido en la } \\
\text { entrevista (escala del } 0 \text { al 100) }\end{array}$ \\
\hline \multirow{5}{*}{$\begin{array}{c}\text { Grupo } \\
\text { intermedio } \\
\text { bajo }\end{array}$} & 6 & belga & 28 \\
\hline & 16 & belga & 40 \\
\hline & 73 & alemana & 43 \\
\hline & 77 & surcoreana & 43 \\
\hline & 75 & alemana & 48 \\
\hline \multirow{8}{*}{$\begin{array}{c}\text { Grupo } \\
\text { intermedio } \\
\text { alto }\end{array}$} & 85 & japonesa & 62 \\
\hline & 8 & checa & 69 \\
\hline & 66 & polaca & 70 \\
\hline & 78 & italiana & 71 \\
\hline & 69 & alemana & 73 \\
\hline & 67 & belga & 73 \\
\hline & 70 & checa & 74 \\
\hline & 76 & china & 75 \\
\hline \multirow{12}{*}{$\begin{array}{c}\text { Grupo } \\
\text { avanzado }\end{array}$} & 79 & estadounidense & 77 \\
\hline & 20 & austriaca & 81 \\
\hline & 15 & francesa & 83 \\
\hline & 84 & italiana & 84 \\
\hline & 86 & inglesa & 85 \\
\hline & 4 & canadiense & 86 \\
\hline & 80 & china & 86 \\
\hline & 68 & alemana & 88 \\
\hline & 82 & alemana & 88 \\
\hline & 83 & estadounidense & 89 \\
\hline & 72 & trinitaria & 93 \\
\hline & 74 & italiana & 97 \\
\hline
\end{tabular}




\subsection{La transcripción de las entrevistas}

Una vez que los estudiantes fueron divididos en tres grupos, de acuerdo con el puntaje obtenido en la evaluación de sus habilidades de escritura descritas en la entrevista, el investigador llevó a cabo las transcripciones con ayuda de tres asistentes. De acuerdo con Knobel y Lankshear (2001) y Zhang y Wildemuth (2009), se buscó que las transcripciones capturaran la mayor cantidad de información posible, aunque estas no se hicieron de forma literal (Ducasse y Brown, 2009). En caso de errores gramaticales o de pronunciación dichos por los entrevistados, se decidió conservarlos y colocar entre corchetes lo que se había querido decir cuando esto no resultaba claro (Bryman, 2012; Galaczi, 2014). Se decidió dejar entre paréntesis información no verbal capturada al momento de la transcripción.

\subsection{El análisis del contenido}

Dado que se partió de un razonamiento inductivo para la identificación y codificación de los datos, se siguió un enfoque de análisis de contenido como el que proponen Zhang y Wildemuth (2009). Entonces, el primer paso consistió en establecer las categorías y unidades de análisis. Las categorías preliminares partieron del guion de la entrevista y de su identificación en las transcripciones (Andréu, 2001; Zhang y Wildemuth, 2009). En cuanto a la unidad de análisis, se eligió el criterio semántico; es decir, el contenido mínimo que proveyera información completa a la pregunta formulada o a otros conceptos emergentes durante la entrevista (Tracy, 2013). Para poder llevar a cabo el análisis, se empleó el software NVivo versión 11.

En el presente estudio, participaron dos codificadores: el investigador y una especialista en investigación cualitativa. Primeramente, el investigador hizo una lectura minuciosa de las 25 entrevistas con el fin de determinar una lista tentativa de categorías de análisis. Paralelamente, la especialista en evaluación cualitativa, quien no estaba familiarizada con el tema de estudio (Neuendorf, 2002 sugiere que, para evitar sesgos, se trabaje con codificadores entrenados pero que no están familiarizados con el propósito de la investigación), se dio a la tarea de hacer una lectura detallada de las entrevistas con el fin de comprender la naturaleza de la investigación. Una vez concluido este proceso, se llevó a cabo una sesión de inducción para la especialista. En esta sesión se discutieron las categorías previamente concebidas por el investigador, pero se dejó abierta la posibilidad de modificarlas. Posteriormente, se codificaron cuatro entrevistas en NVivo de forma independiente por el investigador y la especialista con el fin de ver si las categorías previamente definidas eran suficientes, claras y necesarias. A este proceso se le conoce como codificación de primer ciclo (Saldaña, 2011). Galaczi (2014) sugiere que se valide el esquema de codificación con un estudio piloto para garantizar la validez 
y la confiabilidad del proceso de codificación. Con base en el pilotaje, se redefinieron las categorías y se comenzó nuevamente el proceso de codificación de las 25 entrevistas. Las categorías incluidas se presentan en la Tabla II.

Tabla II. Categorías de análisis y correlación entre codificadores.

\begin{tabular}{|l|c|}
\hline Categorías de análisis & $\begin{array}{c}\text { Kappa } \\
\text { Cohen }\end{array}$ \\
\hline 1. Tiempo en México & .94 \\
2. Tiempo de escribir en un contexto académico en español & .82 \\
3. Autopercepción del nivel de escritura en español & .83 \\
4. Sensaciones al momento de escribir en español & .56 \\
5. Tiempo que le toma escribir en español & .68 \\
6. Toma clases de lengua o manifiesta una opinión al respecto & .83 \\
7. Clases que ha tomado o tomó en un contexto académico en español & .73 \\
8. Idioma de las lecturas asignadas en las clases & .74 \\
9. Idioma de las lecturas optativas o por cuestiones de la investigación & .68 \\
10. Idioma de notas de clase & .69 \\
11. Experiencia con exámenes académicos escritos & .42 \\
12. Experiencia con trabajos académicos escritos & .30 \\
13. Dificultades al escribir en español & .55 \\
14. Influencia de la lengua materna para escribir en español & .78 \\
15. Descripción del proceso de escritura & .71 \\
16. Uso de recursos materiales & .52 \\
17. Edición con ayuda de terceros & .52 \\
18. Retroalimentación que recibe sobre su escritura en español & .57 \\
19. Reflexión sobre qué podría mejorar su escritura académica & .71 \\
20. Otros conceptos no incluidos & .66 \\
\hline & .66 \\
\hline
\end{tabular}

La consistencia entre codificadores se obtuvo mediante el coeficiente de correlación (kappa de Cohen). La correlación muestra qué porcentaje del texto codificado coincide entre los dos o más codificadores. Galaczi (2014) sugiere que el nivel de correlación entre codificadores (kappa de Cohen) sea mayor de 0,6. La Tabla II arriba muestra la correlación kappa de Cohen de los dos codificadores. Como se puede apreciar la correlación más alta fue en la categoría 1 (.94), mientras que la más baja fue en la categoría 11 (.30). El promedio de las correlaciones fue de .66. Sin embargo, estas correlaciones fueron útiles para poder llevar a cabo sesiones de retroalimentación en las cuales se discutieron las posibles causas de discrepancia en la codificación de los datos. Con esta valiosa información, tras el proceso de codificación del investigador y la especialista, se pudo hacer una revisión minu- 
ciosa de aquellas categorías que presentaban bajas correlaciones. Por ejemplo, en el caso de las categorías 11 y 12 (experiencia con trabajos y exámenes escritos) la baja correlación entre codificadores se debió a que uno de ellos no seleccionaba la información cuando los estudiantes asentían que no había tenido exámenes o trabajos escritos, mientras que el otro codificador sí la seleccionaba. Después de discutir dicha discrepancia entre los codificadores, se acordó que se seleccionaría la información incluso cuando los estudiantes afirmaban no haber tenido aún trabajos o exámenes escritos, pues de otra manera se interpretaba que no se había hecho mención alguna al respecto. Una vez que se revisaron estas discrepancias, siguiendo el proceso reflexivo del análisis iterativo del contenido (véase p.ej., Srivastava y Hopwood, 2009), se hizo una segunda revisión de las codificaciones por parte del investigador y la especialista.

\section{RESULTADOS}

A continuación, se hará una descripción detallada de la información recabada en cada uno de los grupos y de acuerdo con las categorías de análisis previamente identificadas. También se presentan algunos ejemplos representativos de la información proporcionada por los estudiantes. Dada la extensión del presente artículo, se ofrecen solamente algunos ejemplos en aquellas categorías en las que se evidenciaron diferencias sustanciales entre los grupos intermedio bajo, alto y avanzado (véase Mendoza, 2017 para mayor información y ejemplos de cada una de las categorías de análisis). De las 19 categorías que se presentarán, solamente la 6 fue de carácter emergente. Esta categoría hacía alusión a la necesidad de tomar o no cursos de lengua. Al final de la descripción de cada uno de los grupos, también se presenta información de la categoría 20, la cual incluía otros conceptos no considerados en el resto de las categorías. Las categorías 16 (uso de recursos materiales para la escritura) y 17 (edición con ayuda de terceros) se fusionaron con la 15 (proceso de escritura), pues se encontraban íntimamente relacionadas entre sí, además de que su integración facilitaba la comprensión del proceso de escritura.

\subsection{Grupo intermedio bajo}

En cuanto a su percepción del nivel de español, reportaron que este era bastante malo, muy malo, malo, suficiente o no tan bueno. De igual manera, sus sensaciones al momento de escribir fueron negativas: frustración, carencia de valor en lo escrito, tristeza, sentirse limitado, o percibirse como tonto. Sólo uno mencionó que se sentía con más confianza; sin embargo, este estudiante tenía apenas cuatro meses en México y poca experiencia de escribir en un contexto académico. 


\section{Categoría 4. Sensaciones al momento de escribir en español}

E6: [...] estoy muy triste, muy frustrada. Siento que no puedo exprimirme [quiso decir expresarme]. [...] No puedo decir lo que quiero decir. Es como si no pudiera mostrar mi valor real.

En cuanto al idioma de las notas de clase, se percibió una fuerte influencia de la lengua materna para tomar notas y escribir información importante para recordar.

\section{Categoría 10. Idioma de las notas de clase}

E77: Mezcla, pero generalmente escribo coreano porque puede escribir más rápido.

Investigador: ¡Ah, escribes en coreano! Entonces, tú escuchas en español al profesor, pero ¿tú tomas tus notas en coreano?

E77: Sí, en coreano, porque... ah... a veces, yo escribo sólo coreano; pero escribo por coreano, porque letras coreanas, este... ¿Cómo se dice?

Investigador: El alfabeto.

E77: Sí, alfabeto, el coreano este... sonido, letras de sonido; pero si yo escribo en español, a veces yo no puedo saber bien como las letras exactamente; por ejemplo, cómo se escribe como... por ejemplo: $b$ o $v$. Como esas cosas, por eso yo escribo por coreano; sólo su sonido, no su sentido.

Investigador: Tú escribes en español usando el alfabeto coreano.

E77: Ah... pero no todo. Yo escribo por coreano; pero solo las palabras, palabras importantes. Si yo no sé su sentido, yo escribo su sonido.

En cuanto al proceso de escritura, se evidenciaron las dificultades que los estudiantes tienen para escribir en español, así como la fuerte dependencia a diccionarios, traductores electrónicos o foros de discusión, aunado a la dificultad de poder discernir entre las distintas acepciones de una palabra. En general, el proceso de escritura se describió como una traducción de la lengua materna al español, o bien escriben en español, pero la estructura del pensamiento se encuentra en otra lengua. Esta forma de escritura, según comentaron, interrumpía el flujo del pensamiento por escrito. La incorporación del material proveniente de distintas fuentes era también incipiente. En cuanto al apoyo de terceras personas, cuando el tutor no era hispanohablante, esto representaba un problema adicional para el estudiante que no contaba con buen nivel de escritura en español. En general, el apoyo de terceras personas era común y muy socorrido por este grupo de estudiantes.

Categorías 15-17. Descripción del proceso de escritura (incluye la fase de 
lectura, planeación, uso de recursos materiales y de la edición del texto con ayuda de terceros)

E77: Ah, primero escribo... como en coreano, esquema y después escribo, y como... explicar, explico en coreano. Escribo otra vez y después escribo en español.

Investigador: Entonces, haces un ejercicio de traducción del coreano al español.

E77: Sí, podemos decirlo así. [...] Es problema de mi manera de hablar. [...] En coreano yo puedo hablar así como nivel alto, sí, pero en español yo no puedo hablar así. Mi oración es, parece como de niño de secundaria, por eso no es tan... como educativo, ¿ajá? Y también es problema de... palabras, porque yo uso diccionario de coreano y español, español y coreano. En mi diccionario dice: Esa palabra significa esto, eso, eso, pero cuando yo escribo mi tesis, de hecho esa palabra no se dice ese sentido, ¿ajá? Por eso siempre yo necesito alguien puede leer otra vez. [...] También yo uso la oración de los libros; este libro una oración, este libro una oración. Por eso un párrafo puede ser, no tener mucha ilación.

\section{Investigador: ¿Utilizas otros recursos?}

E77: Sí, todo: diccionario, también translator de google; sólo google, también yo escribo una oración y escribo en google, para que... saber: ¿las personas están usando esa oración, o no? ¿Esa palabra, o no?

Investigador: ¿Le pides ayuda a alguien para que revise tus textos?

E77: Yo... reviso con mis compañeros primero. [...] mexicanos. Sólo mexicanos, ajá. Porque ellos tienen conocimiento de historia, necesito ese tipo de personas. Pues primero, yo lo veo con mis compañeros, y después yo ah.... Lo revisamos juntos con otros amigos mexicanos, que puedan hablar o leer bien español, pero que no tienen conocimiento de historia. Lo hago dos veces.

\subsection{Grupo intermedio alto}

Sobre su percepción del nivel de escritura en español, comentaron aspectos tanto negativos como positivos. De las sensaciones al momento de escribir, estas fueron diversas. Los sentimientos negativos manifestados fueron de terror, de poca confianza o de tener que hacer el esfuerzo por escribir en español. Los sentimientos positivos fueron de gusto, pese a las dificultades de escribir en español o de dudas sobre lo que se escribe.

\section{Categoría 4. Sensaciones al momento de escribir en español}


E08: Creo que ya, ya es mejor, como el sentimiento que tengo de la parte de escritura es mejor.

En relación con las notas de clase, hubo quienes mencionaron mezclar entre español y otra lengua; sin embargo, hay quienes reconocieron escribir mayoritariamente en español y sólo eventualmente en otro idioma para facilitar la escritura.

\section{Categoría 10. Idioma de las notas de clase}

E66: ¡Ay! Es una mezcla terrible. Es una mezcla de todo, porque también, ¿sabes qué? Depende de los temas, porque algunas cosas... si hay cosas que he aprendido en español, por ejemplo, tomo notas en español, hay cosas que he aprendido... en inglés o polaco depende del tema, depende del tema. Sí, y dependiendo del tema, como te comenté, algunos temas más en inglés, algunos en español. Dependiendo de si se relaciona con algún texto que... he leído anterior, había leído anteriormente en inglés o español, eso influye en que idioma tomo las notas.

El proceso de escritura resultó muy variado en los estudiantes del grupo intermedio alto. Sólo una estudiante, quien puntuó con la nota más baja del grupo, reportó tener un proceso de traducción al español después de escribir su texto en su lengua materna. Los demás estudiantes manifestaron escribir sus textos en español. En algunos casos, mencionaron hacer uso del diccionario para escribir la palabra en español, mientras que, en otros, utilizan su idioma materno intercalado con el español.

Categoría 15. Descripción del proceso de escritura (incluye la fase de lectura, planeación, uso de recursos materiales y de la edición del texto con ayuda de terceros)

E69: Mmm... No, bueno, por ejemplo, si yo escribo un ensayo, pues leo lecturas, ¿no? Entonces, creo que es lo que... lo que sí tarda un poco más es... formar las ideas en español; porque los textos normalmente están en inglés, por ejemplo. Entonces, eso sí; porque a veces no me sale la palabra alemana y tengo problemas de [no se entiende muy bien, parece que dice traducción] y no sé qué; entonces, en alemán como es lengua materna tengo la lectura y sí, sé cómo escribirlo, ¿no? Pero en español, eso creo que sí toma más.

Investigador: Cuando tú lees en español y tienes que escribir en español, y cuando tú lees en inglés y tienes que escribir en español, ¿qué dificultades ves? ¿Es igual de fácil escribir? 
E69: Pues, a mí me gusta más... este..., leer en inglés y escribir en español, porque no tienes tanta... como que no es tan fácil copiar, ¿no? Copiar así, porque yo no quiero tradu... Porque si ya está en inglés, y además así, en un inglés muy bonito, pues no lo voy a copiar, porque eso no se hace; pero como que... sí, es mucho más difícil así, decirlo en otras palabras, si ya las palabras están usadas, ¿no?

Investigador ¿De qué recursos te vales... o cómo lo haces tú para ir construyendo tu texto?

E69: Pues normalmente, sólo pues un diccionario, uso leo, creo que solo hay en alemán online. Porque los diccionarios [se refiere a diccionarios en papel], no, me tardo casi un mes buscando la palabra (risas). Entonces, ahí, porque ahí también tienen este... También ponen, por ejemplo: Eso sólo se dice en España, en otro país, o así, dichos. Y pues también así... ¡Ah, y también uso WordReference! porque ahí también hay como sinónimos, antónimos y eso, pero ya.

A la hora de ir construyendo tu texto, ¿cómo te vienen las ideas?

E69: No. Bueno, yo leo, por ejemplo, leo una lectura o algo, ¿no? Y pues, sí ya me apunto las ideas y los voy acumulando, y este... pero ya todo en español. Entonces, ya como que veo lo que tengo y lo pongo y pongo mis ideas, pero ya todo...

Inevstigador: ¿Y si esas palabras no te vienen en español?

E69: Los busco enseguida, ajá. Cuando me los apunto, es cuando ya enseguida los busco. En eso, normalmente, en el proceso, eso me tarda más bien como... De todo, como que... poner todas las ideas así, en la compu y pues ya en español. Como fichas, eso lo hago, pero en la compu.

Investigador: ¿Le pides ayuda a alguien?

E69: Me da pena (risas). Este... bueno... para, por ejemplo, para mis cartas de motivación, cosas así, sí se los pido a mis amigos, pero... así para un trabajo escrito... Es que no me parece tan... como que no es una tesis, ¿no? Entonces, no me parece tan importante para que lo tengan que leer, porque normalmente son como veinte páginas y, si a mí alguien me da algo para leerlo, yo siempre estoy así de: ¡Ay, bueno! Pues sí lo hago, pero... sí es muy pesado.

\subsection{Grupo avanzado}

La autopercepción del nivel de escritura en español fue muy diversa. Hubo quienes afirmaron que su nivel era principiante, más bajo que en su lengua materna, intermedio, bueno, bastante bueno y avanzado. Quienes afirmaron que su nivel 
era avanzado, matizaron que este podía mejorar. Con respecto a las sensaciones que reportaron al momento de escribir, todas fueron positivas. Solo una estudiante mencionó que se sentía chistosa al escribir en español, pues sentía que utilizaba estructuras muy enredadas. Los estudiantes del grupo avanzado sentían que podían expresar bien sus ideas, tenían confianza y escribían con placer y comodidad en español. Sin embargo, algunos también manifestaron que esta confianza no siempre era positiva, pues podía conducir a errores. De igual forma, dos estudiantes manifestaron que el placer por escribir en español no se comparaba aún con el gusto por escribir en lengua materna.

\section{Categoría 4. Sensaciones al momento de escribir en español}

E84: Absolutamente con confianza, pero eso eh... no creo que sea totalmente positivo porque eso me lleva a... hacer muchos errores.

Sobre el idioma en que toman sus notas de clase, en general, mencionaron escribir todo en español, aunque algunos estudiantes reportaron que de vez en cuando introducían algunas palabras o notas en otra lengua, esto con el fin de recordar conceptos o ideas importantes.

\section{Categoría 10. Idioma de notas de clase}

E86: Ahm... Ya siempre en español, siempre en español. A veces, si la lectura está en inglés, hago mis notas en inglés; por siempre refleja el idioma de la lectura ¿no? [...] Pero, en general escribo en español, siempre. Hasta que a veces las lecturas están en inglés y escribo mis notas en español, porque voy a tener que hablar sobre ellas en español.

En relación con el proceso de escritura, los estudiantes del grupo avanzado reportaron en general un excelente dominio de la escritura académica en español. Evidenciaron que el proceso de escritura reflejaba ya un pensamiento y procesamiento de la información en español. De igual forma, algunos de ellos enfatizaron que previo al proceso de escritura había un periodo de lectura con formulación de conceptos e ideas de cómo planificar la escritura incluso desde la lectura. La gama de estrategias que desplegaron para escribir fue variada; es decir, dependiendo de su conveniencia, no siempre escribían todo en español, sino que en ocasiones se apoyaban de su lengua materna L2 para escribir con mayor rapidez y darle fluidez a su discurso. Naturalmente, reconocieron que el proceso de construcción del conocimiento era gradual y sufría considerables modificaciones desde la planeación hasta la consecución final del escrito. Los recursos materiales que empleaban 
para escribir eran generalmente diccionarios y traductores electrónicos, aunque la forma de emplearlos parecía estar dirigida más hacia un sistema eficiente de escritura que no siempre implicaba escribir todo en español durante el proceso de escritura, sino que también podía ser durante la edición del texto. Asimismo, se percibió una preferencia por la fluidez en el flujo del pensamiento plasmado en la escritura más que en la preferencia de escribir en una lengua en particular. Una vez que contaban con un escrito preliminar, los estudiantes reportaron acudir a diversas personas para revisar sus textos, en general amistades o parejas, pero también manifestaron que esta ayuda se encontraba más bien focalizada a ciertos aspectos del texto.

Categoría 15-17. Descripción del proceso de escritura (incluye la fase de lectura, planeación, uso de recursos materiales y de la edición del texto con ayuda de terceros)

\section{Investigador: ¿¿No se te dificulta escribir en español si lees en inglés?}

E20: Primero leo las cosas que necesito. Eh... pongo... empiezo con una referencia que sé que es importante para el trabajo, algo así y luego lo que me pasa mucho es que pongo una idea, algo como más claro y en ese proceso se me ocurre algo y lo pongo más abajo. Entonces, cuando termino la idea, paso a la siguiente y puede ser un ir y venir entre lo que he leído y los puntos que necesito tocar. Después lo releo para ver si estoy repitiendo la información.

Investigador: ¿Cuándo escribes en la computadora, todo es en español? E20: Sí, a menos que leí algo en inglés, entonces lo pongo en inglés y luego lo traduzco. Por ejemplo, ahora que estoy leyendo en alemán un libro, no sé si poner mis notas en alemán o español, pero hago lo que me viene más rápido. Los conceptos, como estoy hablando más en español, los hago en español. Si no la escribo en inglés, se me va la idea. Escribo un poquito más en inglés y luego la traduzco en español. Porque después siento mucho que se me va la idea. Si regreso a una nota, me cuesta trabajo recordar a qué se refería. Siempre es lo que me llega más rápido. Si lo conecto con una idea que ya tengo, aunque el artículo está en inglés, la escribo en español. Investigador: ¿Utilizas diccionarios u otros recursos para escribir? E20: Más que nada lo que hacía era buscar palabras o expresiones académicas en Google. $\mathrm{O}$, es que no me acuerdo bien, pero también en artículos iba identificando como empiezan, como concluyen, ciertas expresiones. 


\section{Investigador: ¿Le pides a alguien que revise tus textos?}

E20: [...] En el escrito, poco a poco le iba dando mis trabajos a una amiga y ella me lo corregía. Más que nada me decía de la gramática. [...] Por ejemplo, mi tutor también me iba diciendo cosas. [...] sé que necesito que me chequen el trabajo, porque yo no lo noto [refiriéndose a cosas que escribe que no son claras para los lectores, pero que no sabe cómo autocorregir].

De las categorías previamente determinadas, solamente hubo una que resultó emergente. La categoría 6 en la que los estudiantes expresaban su opinión o necesidad de tomar clases de español. Otros conceptos que valdría la pena explorar en futuras investigaciones se sistematizaron en la categoría 20. Algunos de estos conceptos fueron: las necesidades de escritura para estudiantes de posgrado que se encuentran en intercambios académicos cortos y la proyección del uso de la escritura en el futuro.

\section{DISCUSIONES, IMPLICACIONES Y FUTURAS INVESTIGACIONES}

En primer lugar, cabe destacar que, si bien la correlación entre los resultados de la PE del EXELEAA y la evaluación de la entrevista fue fuerte $(r=0,72)$, de los cinco estudiantes que en la entrevista demostraron habilidades y estrategias de escritura no aptas para el ámbito académico, solo el estudiante 73 recibió una nota no aprobatoria en la PE del EXELEAA. Esto significa que quizás sería necesario revisar los puntos de corte de la PE del EXELEAA a la luz de las entrevistas o implementar otros puntos de corte complementarios (p.ej. Kenyon y Römhild, 2014). Adicionalmente, haciendo eco a los estudios de Fox (2004), las autoridades de cada posgrado deberían darle seguimiento a los estudiantes que obtuvieron puntuaciones bajas en el examen con el fin de ofrecerles talleres o cursos de escritura académica, o cursos adicionales de lengua, en caso de ser necesario.

Ahora bien, en cuanto al análisis de las entrevistas, con el fin de poder establecer la relación entre las distintas categorías de análisis del presente estudio, se muestra un esquema de relación de las mismas. La Figura 1 muestra el esquema de relación de los componentes asociados a las habilidades y estrategias de escritura académica. Este esquema se encuentra conformado por un componente principal: el proceso de escritura académica, y por tres componentes más asociados a él: la autopercepción de la escritura, aspectos que favorecen la escritura y reflexiones sobre qué podría mejorar la escritura. 


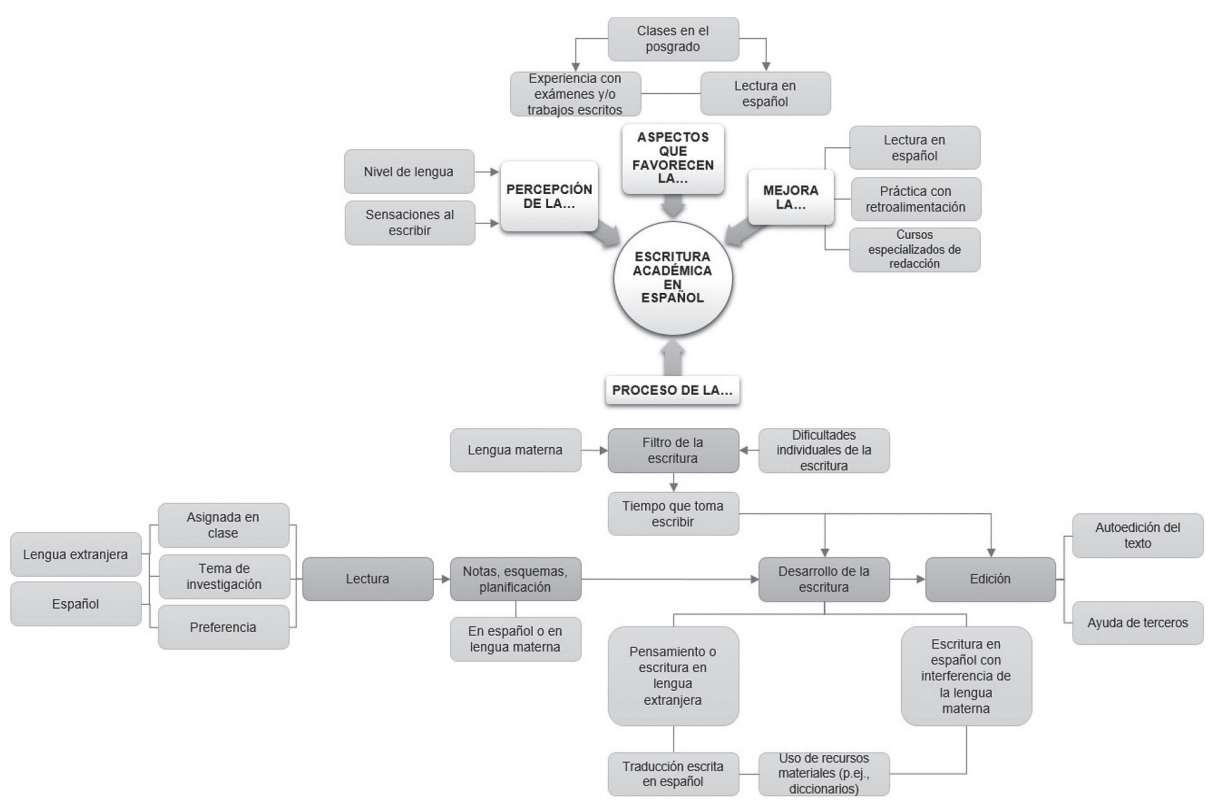

Figura 1. Esquema de relación de los componentes asociados a las habilidades de escritura académica en español como LE. (Desarrollado por el investigador).

En cuanto al proceso de escritura, las habilidades y estrategias se evidencian desde la toma de notas de lecturas y de clase, las cuales posteriormente se ven plasmadas en notas más elaboradas, esquemas y fichas que luego les servirán para redactar sus textos académicos. Los estudiantes menos proficientes tienden tomar notas en lengua materna, mientras que los estudiantes con habilidades más desarrolladas lo hacen en su lengua materna solo de manera estratégica. Estos hallazgos refuerzan estudios como los de Cassany (2006) y Cassany y Morales (2008) que perciben el proceso de lectoescritura como indisociable. El idioma de las lecturas también juega un papel imprescindible en el desarrollo de las habilidades de escritura, de ahí que la lectura en la lengua meta favorezca el desarrollo léxico, morfológico y sintáctico del lenguaje académico escrito. De hecho, los estudiantes que leen mayoritariamente en otra lengua distinta al español presentan mayores dificultades para desarrollar sus habilidades de escritura académica en español. En cuanto a la selección del idioma de las lecturas, esta responde a diversas causas: el tema de investigación, la facilidad de encontrar material disponible, conveniencia, practicidad o simplemente preferencia.

El proceso de escritura en computadora comienza tras la lectura y una vez que los estudiantes cuentan con una planificación de la escritura. Dependiendo del nivel de lengua del estudiante o de sus preferencias de escritura, el uso de recursos materiales (p.ej., diccionarios, traductores, foros de discusión) es simultáneo a la 
escritura o posterior durante la fase de edición (Singh, 2017). En este momento, la lengua materna y las dificultades individuales al escribir en español repercuten directamente en el tiempo que toma este desarrollo de la escritura. En cuanto mayores son las dificultades del uso de la lengua escrita en español y más distante es la lengua materna, mayor es el tiempo que le toma escribir al estudiante (incluso más del doble de lo que le tomaría hacerlo en lengua materna). Por el contrario, este tiempo disminuye conforme incrementa el nivel de dominio de la lengua escrita y la lengua materna se acerca más al español.

En relación con el desarrollo de la escritura, de manera similar a los hallazgos de Sasaki (2014), los estudiantes que no cuentan con habilidades de escritura apta para el ámbito académico demuestran un proceso de escritura prácticamente de traducción; ya sea de manera sincrónica (i.e., se piensa primero en otra lengua y se intenta traducir al español simultáneamente), o diacrónica (i.e., escriben primero el texto en lengua extranjera y después traducen el texto al español). Estos estudiantes también son dependientes de los recursos materiales para escribir en español (diccionario, foros de discusión o traductores electrónicos) pues sin ellos, su proceso de escritura se ve imposibilitado (véase p.ej., Singh, 2017, p. 632). Otra de las dificultades con el uso del diccionario es que no logran comprender el uso de las distintas acepciones de una palabra. Finalmente, los estudiantes menos proficientes no cuentan con estrategias eficientes de ayuda en la edición de sus textos, ya sea porque no la solicitan -quizás porque saben el trabajo que implica revisar textos completos- o porque la solicitan en todo momento para la revisión completa de sus textos (véase también Fox, 2004).

Los integrantes del grupo intermedio alto cuentan con un proceso de escritura en español más desarrollado. Escriben sus textos en español, aunque algunos intercalan palabras o frases en otro idioma. En general, su proceso de escritura no es tan fluido porque su pensamiento, si bien es mayoritariamente en español, presenta algunas lagunas lingüísticas (i.e., gramaticales, sintácticas, léxicas) que les impiden expresar sus ideas por escrito de forma continua y sin interrupciones. Al no encontrar la palabra, frase o estructura gramatical, la escriben en otro idioma, pero el flujo de pensamiento se ve parcialmente interrumpido. También hay quienes desean escribir todo su texto de una vez en español, para lo cual invierten tiempo de búsqueda en traductores electrónicos, que va en detrimento del flujo de ideas. Aparentemente este grupo no es tan dependiente del diccionario/traductor electrónico, pero lo ocupan con relativa frecuencia para tener más riqueza léxica. En cuanto al proceso de edición de sus textos, los estudiantes de este grupo son más selectivos y solicitan la revisión de ciertas partes del texto. Esto demuestra también que están más conscientes de dónde tienen problemas para expresarse.

Finalmente, el grupo avanzado denota un desarrollo de escritura fluido. Su pensamiento en español se encuentra mucho más consolidado. De vez en cuando escriben alguna palabra en otra lengua o emplean traductores electrónicos para 
buscar sinónimos o léxico que resulte más académico o especializado. En general, cualesquiera de las estrategias que emplean va en favor del flujo del pensamiento (véase p.ej., Weigle, 2002, 2010). Es decir, con tal de no perder una idea, pueden mezclar información en español o en otra lengua. Por último, en relación con el proceso de edición de textos, estos estudiantes mostraron un alto grado selectivo y sofisticado de edición que involucra a distintos actores (p.ej., el tutor, amigos, compañeros de clase o parejas sentimentales).

En cuanto a la percepción de la escritura, los estudiantes menos proficientes consideran que su nivel de escritura no es bueno, además de describir experiencias y sensaciones negativas al momento de escribir en español. Fox (2004) afirma que es importante darles seguimiento a los estudiantes que presentan problemas para expresarse en la lengua meta, pues esta carencia incide en la desmotivación y puede conducir a la deserción académica. Por el contrario, los estudiantes más aptos perciben que su nivel de escritura es adecuado y describen sensaciones de agrado, placer y confianza al escribir en español, aunque reconocen que no es lo mismo que escribir en lengua materna. En relación con los aspectos que favorecen la escritura académica en español, tomar clases en el posgrado y tener que desarrollar trabajos escritos a lo largo del semestre académico les ayuda significativamente a los estudiantes a mejorar paulatinamente su escritura académica. En este sentido, los alumnos de doctorado generalmente se ven desfavorecidos, puesto que generalmente no toman clase, sino que su escritura académica se limita a la redacción de la tesis. Finalmente, sobre qué podría mejorar la escritura académica, los estudiantes de todos los niveles destacan que esta se ve ampliamente favorecida a través de la lectura y de la práctica, siempre y cuando haya corrección y retroalimentación individual de los textos. De hecho, es a través de la lectura en español que se adquiere vocabulario, expresiones y frases académicas, además de que también se aprenden estructuras lingüísticas y convenciones de escritura propias del ámbito académico. En cuanto a la corrección, esta puede ser por parte de docentes o tutores del posgrado, compañeros de clase o provenir del apoyo en clases de redacción enfocadas específicamente a la revisión de textos académicos.

\section{CONCLUSIONES}

Las instituciones hispanas deben garantizar que sus prospectos no nativos hablantes cuentan con las habilidades de escritura suficientes para hacer frente a las exigencias académicas de sus posgrados. Por esta razón, resulta indispensable evaluar el uso de la lengua de dichos prospectos universitarios y garantizar que el examen mide eficazmente lo que se pretende medir. Una manera de dar cuenta de las implicaciones de un examen en la vida de los examinados es conduciendo estudios de extrapolación. En el ámbito académico, estos estudios permiten, por una parte, 
establecer la relación que subyace entre el dominio de la lengua demostrado en un examen y el contexto de uso. Por otro lado, los estudios de extrapolación del uso de la lengua escrita permiten que se indague en las habilidades y estrategias que emplean los estudiantes no hispanohablantes durante su proceso de escritura y edición de textos académicos.

\section{REFERENCIAS}

Andréu, J. (2001). Las técnicas de análisis de contenido: Una revisión actualizada. Documento de trabajo, S2001/03, Centro de estudios andaluces.

Angulo, N. (2013). La cita en la escritura académica. Innovación Educativa, 13 (63), 96-116.

Bachman, L., y Palmer, A. (1996). Language Testing in Practice. Oxford University Press.

Bachman, L., y Palmer, A. (2010). Language assessment in practice: Developing language assessments and justifying their use in the real world. Oxford: Oxford University Press.

Bereiter, C., y Scardamalia, M. (1987). The psychology of written composition. Hillsdale, NJ: Lawrence Erlbaum.

Bryman, A. (2012). Social Research Methods. (4ta. Edición). Nueva York: Oxford University Press.

Carciu, O. M. (2009). An intercultural study of first-person plural references in biomedical writing. Ibérica: Revista de la Asociación Europea de Lenguas para Fines Especificos (AELFE), 18, 71-92.

Carlino, P. (2003). Alfabetización académica: Un cambio necesario, algunas alternativas posibles. Educere. Investigación, 6 (09), 409-417.

Carlino, P. (2013). Alfabetización académica diez años después. Revista Mexicana de Investigación Educativa, 18 (57), 355-381.

Cassany, D. (2006). Taller de textos. Leer, escribir y comentar en el aula. Barcelona: Paidós

Cassany, D y Morales, O. (2008). Leer y Escribir en la Universidad: Hacia la lectura y la escritura crítica de géneros científicos. Disponible:http://www.saber. ula.ve/bitstream/123456789/16457/1/leer_universidad.pdf.

Castelló, M. (2014). Los retos actuales de la alfabetización académica: estado de la cuestión y últimas investigaciones. Enunciación, 19 (2), 346-365.

Castelló, M. e Iñesta, A. (2012). Texts as artifacts-in activity: Developing authorial identity and academic voice in writing academic research papers. En M. Castelló y C. Donahue (Eds.). University writing: Selves and Texts in Academic Societies. Londres: Emerald Group Publishing Limited.

Chang, S. H., Wu, R. Y., y Weir, C. J. (2014). Examining the context and cogniti- 
ve validity of the GEPT Advanced Writing Task 1: A comparison with real-life academic writing tasks. LTTC-CRELLA Collaboration Project RG-03. Taiwan. Dörnyei, Z. (2007). Research Methods in Applied Linguistics: Quantitative, Qualitative, and Mixed Methodologies. Oxford: Oxford University Press.

Ducasse, A.M., y Brown, A. (2009). Assessing paired orals: Raters' orientation to interaction. Language Testing, 26 (3), 423-443.

Encinas, F., y Keranen, N. (2010). Curriculum redesign and ELT university sector writing: responding to global factors interpreted by local players. En M. Perales (Ed.) Literacy in Mexican Higher Education: Texts and Contexts. Benemérita Universidad Autónoma de Puebla. México.

Flores, M. A. (2014). La competencia comunicativa escrita de los estudiantes de ingeniería y la responsabilidad institucional. Innovación Educativa, 14 (65), 44-59.

Fox, J. (2004). Test decisions over time: tracking validity. Language Testing, 21 (4), 437-465.

Fulcher, G. (2010). Practical Language Testing. London: Hooder Education/Routledge.

Galaczi, E.D. (2014). Content Analysis. En A. Kunnan. (Ed.), The Companion to Language assessment. Vol. 3. Chapter 78 (pp. 1325-1339). Oxford, United Kingdom: Wiley-Blackwell.

González, J. (2014). Los niveles de conocimiento. El Aleph en la innovación curricular. Innovación Educativa, 14 (65), 133-141.

Hayes, J. R. (1996). A New framework for understaing cognition and affect in writing. En M. Levy y S. Ransdell, The Science of Writing (pp. 1-27). New Jersey: Lawrence Erlbaum Associates.

Hayes, J., y Flower, L. (1980). Identifying the organization of writing processes. En L. Gregg y E. Steinberg, (Eds.) Cognitive processes in writing: An interdisciplinary approach (pp. 3-30). Hillsdale, NJ: Lawrence Erlbaum.

Hewitt, E., y Felices, A. (2010). Academic style and format of doctoral theses: The case of the disappearing discussion chapter. Ibérica: Revista de la Asociación Europea de Lenguas para Fines Especificos (AELFE), 19, 19-140.

Kane, M. (1992). An argument-based approach to validation. Psychological Bulletin, 112, 527-535.

Kane, M. (2002). Validating high-stakes testing programs. Educational Measurement: Issues and Practice, 21 (1), 31-41.

Kane, M. (2006). Validation. En R. Brennan (Ed.). Educational measurement (pp. 17-64). Westport: American Council on Education and Praeger.

Kane, M. (2013). Validating the Interpretations and Uses of Test Scores, Journal of Educational Measurement, 50 (1), 1-73.

Kane, M., Crooks, T., y Cohen, A. (1999). Validating measures of performance. Educational Measurement: Issues and Practice, 18 (2), 5-17. 
Kellog, R. (1996). A model of working memory in writing. En M. Levy y S. Ransdell. (Eds.) The Science of writing: Theories, methods, individual differences, and applications (pp. 57-72). Mahwah, NJ: Lawrence Erlbaum Associates.

Kenyon, D.M., y Römhild, A. (2014). Standard Setting in Language Testing. En A. Kunnan. (Ed.), (pp. 944-961). The Companion to Language assessment. Vol. 2. Chapter 57. Oxford, United Kingdom: Wiley-Blackwell.

Knobel, M., y Lankshear, C. (2001). Maneras de Ver: El Análisis de Datos en Investigación Cualitativa. Morelia: Instituto Michoacano de Ciencias de la Educación.

Knoch, U. (2011). Rating scales for diagnostic assessment of writing: What should they look like and where should the criteria come from? Assessing Writing, 16, 81-96.

Krippendorff, K. (2004). Content analysis: An introduction to its methodology (2a edic.). Londres: Sage.

Leki, I., Cumming, A., y Silva, T. (2008). A Synthesis of Research on Second Language Writing in English. New York: Routlegde.

Lindlof, T. R., y Taylor, B. C. (2011). Qualitative communication research methods. ( $3^{\text {ra }}$ edic.). Thousand Oaks, CA: Sage.

Mann, S. (2016). The Research Interview. Reflective Practice and Reflexivity in Research Processes. Warwick: Palgrave Macmillan.

Mendoza, A. (2014). Las prácticas de evaluación docente y las habilidades de escritura requeridas en el nivel posgrado. Innovación Educativa, vol. 14, 66 (3), $147-175$.

Mendoza, A. (2015a). La selección de las tareas de escritura en los exámenes de lengua extranjera destinados al ámbito académico. Revista Nebrija de Lingüística Aplicada a la Enseñanza de Lenguas, 18, 106-123.

Mendoza, A. (2015b). La validez en los exámenes de alto impacto. Un enfoque desde la lógica argumentativa. Perfiles Educativos, 37 (149), 169-186.

Mendoza, A. (2017). El Argumento de validación de la prueba escrita del examen de español como lengua extranjera para el ámbito académico de la UNAM. Tesis doctoral, Universidad Nacional Autónoma de México.

Mendoza, A. y Knoch, U. (2018). Examining the validity of the analytic rating scale for a Spanish test for academic purposes using the argument-based approach to validation. Assessing Writing, 35, 41-55.

Neuendorf, K.A. (2002). The content analysis guidebook. Thousand Oaks, C.A.: Sage.

Riach, K. (2009). Exploring participant-centred reflexivity in the research interview. Sociology, 43 (2), 356-370.

Saldaña, J. (2011). Fundamentals of qualitative research. Nueva York, NY: Oxford University Press.

Sasaki, M. (2014). Hypothesis Generation and Hypothesis Testing: Two Com- 
plementary Studies of EFL Writing Processes. En P. K. Matsuda y T. Silva, (Eds.) (2a edic.). Second Language Writing Research. Perspectives on the Process of Knowledge Construction. Nueva York y Londres: Routledge.

Singh, M. M. (2017). International EFL/ESL Master Students' Adaptation Strategies for Academic Writing Practices at Tertiary Level. Journal of International Students, 7 (3), 620-643.

Srivastava, D. y Hopwood, D. (2009). A Practical Iterative Framework for Qualitative Data Analysis. International Journal of Qualitative Methods, 8 (1), 76-84. Tracy, S. J. (2013). Qualitative research methods: collecting evidence, crafting analysis, communicating impact. Chichester, West Sussex, United Kingdom: WileyBlackwell.

Turner, D. W. (2010). Qualitative Interview Design: A practical guide for novice investigators. The Qualitative Report, 15 (3), 754-760.

Vine, A., y Ferreira, A. (2016). Propuesta de un modelo para una prueba con fines específicos académicos en ELE. Literatura y Lingüistica, 33, 369-390.

Weigle, S. (2002). Assessing writing. Cambridge: Cambridge University Press.

Weigle, S. (2010). Validation of automated scores of TOEFL iBT tasks against non-test indicators of writing ability. Language Testing, 27 (3), 335-353.

Weir, C. (2005). Language Testing and Validation: An Evidence-Based Approach. Basingstoke: Palgrave Macmillan.

Xi, X. (2010). How do we go about investigating test fairness? Language Testing, 27 (2), 147-170.

Zhang, Y., y Wildemuth, B. (2009). Qualitative analysis of content. En B. Wildemuth. (Ed.), Applications of Social Research Methods to Questions in Information and Library Science (pp. 308-319). Westport, CT: Libraries Unlimited.

Zhizhko, E. A. (2014). La enseñanza de la escritura y lectura de textos académicos a los futuros investigadores educativos. Innovación Educativa, 14 (65), 99-113. 


\begin{abstract}
ANEXO 1
Guion de entrevista semiestructurada para estudiantes de posgrado no hispanohablantes de la UNAM
\end{abstract}

OBJETIVO: Investigar cuáles son las habilidades y estrategias de los estudiantes de posgrado no hispanohablantes al momento de escribir en el contexto universitario.

Percepción sobre el dominio de la escritura académica

¿Cuánto tiempo tienes escribiendo en español en un contexto universitario?

¿Cómo consideras que es tu nivel de escritura en la universidad? (por ejemplo: malo, suficiente, bueno, muy bueno, excelente)

¿Cómo te sientes escribiendo en español? (por ejemplo: frustrado, con temor, con desconfianza, con confianza, emocionado, seguro)

¿Cuánto consideras que te tardas en escribir en español? (por ejemplo: lo normal, más de lo normal, mucho más de lo normal, muchísimo más de lo normal)

\title{
Factores contextuales que facilitan o dificultan la escritura académica
}

¿Tomas clases en este momento?

¿En qué idioma está el material que lees normalmente?

¿En qué idioma tomas tus notas? ¿Son a mano o computadora?

¿Tu lengua materna, facilita o dificulta tu proceso de escritura en español?

La escritura en el contexto académico

\section{EXÁMENES}

¿Tienes o has tenido exámenes escritos en clase?

¿Qué dificultades encuentras al momento de escribir un examen en clase? 


\section{TRABAJOS}

¿Te han evaluado con trabajos escritos?

¿Cuál consideras que es tu mayor dificultad al escribir en español? (por ejemplo: gramática, nexos, vocabulario, coherencia, cohesión, tipología textual).

¿Qué trabajo académico te ha costado más escribir y por qué? (por ejemplo: hacer resumen de artículo o libro, abstract, ensayo, monografía, resumen de artículo en inglés)

¿Cómo es tu proceso de escritura?

\section{Recursos empleados durante el proceso de escritura académica}

¿Qué recursos empleas cuando escribes? (por ejemplo: diccionario, tesauros, traductores, pides ayuda a alguien).

¿Qué tipo de retroalimentación recibes de tus profesores? ¿Es suficiente?

¿Qué crees que te ayudaría a mejorar tu escritura académica? 


\section{ANEXO 2}

Rúbrica para evaluar las habilidades y estrategias de escritura académica de los estudiantes no hispanohablantes del posgrado de la UNAM

\begin{tabular}{|c|c|}
\hline \multicolumn{2}{|l|}{$\begin{array}{l}\text { Según lo que escucha usted en la grabación, registre la información que proporcionan } \\
\text { los candidatos respecto a los siguientes rubros: }\end{array}$} \\
\hline \multicolumn{2}{|l|}{ ¿Cuánto tiempo tiene escribiendo en un contexto académico en español? } \\
\hline Menos de un mes & 0 \\
\hline Entre 1 y 6 meses & 1 \\
\hline Entre 6 meses y 1 año & 2 \\
\hline Más de un año & 3 \\
\hline No hay suficiente información para evaluar su desempeño en este rubro. & NA \\
\hline \multicolumn{2}{|l|}{ ¿Qué sensaciones describe al momento de escribir en español? } \\
\hline Muy negativas & 0 \\
\hline Negativas & 1 \\
\hline Positivas o neutras & 2 \\
\hline Muy positivas, aunque reconoce que puede mejorar. & 3 \\
\hline No hay suficiente información para evaluar su desempeño en este rubro. & NA \\
\hline \multicolumn{2}{|l|}{ ¿Cuánto tiempo requiere para escribir en español? } \\
\hline $\begin{array}{l}\text { Aún no escribe en un contexto académico o el tiempo que le toma escribir es } \\
\text { excesivamente mayor al que le tomaría hacerlo en su lengua maternal. }\end{array}$ & 0 \\
\hline $\begin{array}{l}\text { El tiempo que le toma escribir en español es considerablemente mayor al que le } \\
\text { toma hacerlo en lengua materna (el doble). }\end{array}$ & 1 \\
\hline $\begin{array}{l}\text { El tiempo que le toma escribir en español es un poco más que lo que le toma } \\
\text { en lengua materna. }\end{array}$ & 2 \\
\hline $\begin{array}{l}\text { El tiempo que le toma escribir es prácticamente igual que en lengua materna o } \\
\text { en la lengua en que está acostumbrado a escribir. }\end{array}$ & 3 \\
\hline No hay suficiente información para evaluar su desempeño en este rubro. & NA \\
\hline \multicolumn{2}{|l|}{ ¿Ha tomado o toma clases en su posgrado? } \\
\hline Ninguna & 0 \\
\hline Por lo menos una & 1 \\
\hline Dos o tres & 2 \\
\hline Más de tres & 3 \\
\hline No hay suficiente información para evaluar su desempeño en este rubro & NA \\
\hline \multicolumn{2}{|l|}{ ¿Toma clases de lengua o considera que las necesita? } \\
\hline Toma clases de lengua. & 0 \\
\hline No toma clases de lengua y reconoce que las necesita. & 1 \\
\hline
\end{tabular}




\begin{tabular}{|l|l|}
\hline No toma clases, aunque considera que le ayudarían cursos de escritura. & 2 \\
\hline No toma clases y considera que no las necesita. & 3 \\
\hline $\begin{array}{l}\text { No hay suficiente información para evaluar su desempeño en este rubro. } \\
\text { ¿Aproximadamente qué cantidad de material leído por el candidato en sus cursos de } \\
\text { posgrado está en español? }\end{array}$ & NA \\
\hline Ningún material (sólo se leen textos en otro idioma). & 0 \\
\hline La mitad. & 1 \\
\hline Más de la mitad. & 2 \\
\hline Prácticamente todo. & 3 \\
\hline No hay suficiente información para evaluar su desempeño en este rubro. & NA \\
\hline
\end{tabular}

\begin{tabular}{|l|l|}
\hline \multicolumn{2}{|c|}{ ¿Si el candidato hace lecturas extras, ¿’opta por hacerlas en español? } \\
\hline Nunca, porque considera su nivel de lengua insuficiente. & 0 \\
\hline $\begin{array}{l}\text { En pocas ocasiones, porque no existe material pertinente para su investigación } \\
\text { o porque prefiere lecturas en lengua extranjera. }\end{array}$ & 1 \\
\hline $\begin{array}{l}\text { En varias ocasiones, dependiendo de la pertinencia para su investigación. No le } \\
\text { importa leer en una u otra lengua. }\end{array}$ & 2 \\
\hline $\begin{array}{l}\text { En múltiples ocasiones, porque las utiliza como estrategia para favorecer su } \\
\text { escritura. Prefiere lecturas en español. }\end{array}$ & 3 \\
\hline No hay suficiente información para evaluar su desempeño en este rubro. & NA \\
\hline \multicolumn{2}{|l|}{ ¿Elidato utiliza el español para tomar notas en la clase? } \\
\hline Nunca, escribe la mayoría de sus notas en lengua materna o en alguna otra. & 0 \\
\hline $\begin{array}{l}\text { En pocas ocasiones, pero más bien mezcla sus notas en lengua materna y } \\
\text { español. }\end{array}$ & 1 \\
\hline $\begin{array}{l}\text { En varias ocasiones, salvo cuando no comprende conceptos importantes en } \\
\text { español o no sabe cómo traducirlos. }\end{array}$ & 2 \\
\hline En múltiples ocasiones. & 3 \\
\hline No hay suficiente información para evaluar su desempeño en este rubro. & NA \\
\hline \multicolumn{1}{|c|}{ ¿Qué experiencia tiene escribiendo en un contexto académico (trabajos y } \\
\hline $\begin{array}{l}\text { Se encuentra ampliamente familiarizado con los trabajos y/o exámenes escritos } \\
\text { en el ámbito universitario. }\end{array}$ & 3 \\
\hline No hay suficiente información para evaluar su desempeño en este rubro. & NA \\
\hline Tiene muy poca experiencia con trabajos y exámenes escritos en español. & 1 \\
\hline Ha escrito algunos trabajos académicos y/o quizás también exámenes. & 2 \\
\hline
\end{tabular}




\begin{tabular}{|l|l|}
\hline \multicolumn{2}{|c|}{ ¿Cómo describe el candidato su proceso de escritura en español? } \\
\hline Insuficiente para producir textos en español. & 0 \\
\hline Poco eficiente porque se basa en la traducción de la lengua materna al español. & 1 \\
\hline $\begin{array}{l}\text { Adecuado, aunque los huecos léxicos, gramaticales o sintácticos en ocasiones } \\
\text { entorpecen el flujo de sus ideas. }\end{array}$ & 2 \\
\hline $\begin{array}{l}\text { Fluido, aunque con algunos titubeos en la gramática o vocabulario que } \\
\text { prácticamente no impiden el flujo de sus ideas. }\end{array}$ & 3 \\
\hline No hay suficiente información para evaluar su desempeño en este rubro. & NA \\
\hline \multicolumn{2}{|c|}{ ¿Con qué frecuencia se apoya en recursos materiales (traductores, diccionarios o } \\
\hline $\begin{array}{l}\text { Siempre y sin estrategia alguna, pues su nivel de lengua no es suficiente para } \\
\text { utilizarlos de manera adecuada. }\end{array}$ & 0 \\
\hline En múltiples ocasiones y con pocas estrategias de uso. & 1 \\
\hline En varias ocasiones, pero con algunas dificultades de uso. & 2 \\
\hline Esporádicamente (porque no los requiere) y los usa de manera eficiente. & 3 \\
\hline No hay suficiente información para evaluar su desempeño en este rubro. & NA \\
\hline \multicolumn{2}{|c|}{ ¿Cuenta con lectores (amigos, tutor, compañeros, etc.) que retroalimenten sus } \\
\hline Con ninguno, porque no cuenta con estrategias de apoyo. & 0 \\
\hline Con pocos y la retroalimentación no es suficiente. & 3 \\
\hline Con varios lectores que le permiten mejorar su escritura. \\
\hline $\begin{array}{l}\text { Con diversos lectores. Cuenta con sofisticadas estrategias de apoyo } \\
\text { dependiendo del tipo de retroalimentación que necesita. }\end{array}$ \\
\hline No hay suficiente información para evaluar su desempeño en este rubro. \\
\hline
\end{tabular}

